\title{
ICT Integrated Learning: Using Spreadsheets as Tools for e-Learning, A Case of Statistics in Microsoft Excel
}

\author{
Nchimunya Chaamwe and Langstone Shumba
}

\begin{abstract}
The integration of ICTs in the teaching and learning curricula is the apparent goal for initiatives to improve education in schools. Most Schools however, look for specialised educational software and hardware to achieve this integration. This paper proposes the use of a widely readily available tool to achieve the same integration that other specialized tools would achieve. Over the years the application area for spreadsheets has multiplied. It is software that is becoming more pronounced in the teaching and learning of pupils and students. A Spreadsheet can be used as a powerful learning tool for both primary and secondary school pupils. Spreadsheets offer concrete ways to explore abstract concepts in mathematics and other subjects. Spreadsheets contain a variety of formulas, which can be used in teaching mathematics. These formulas can be generalized, so that students can see how the outcome is changed when one of the variables is altered. Apart from using ready-made formulas pupils can make up their own formulas to manipulate numbers. This paper proposes the use of Ms Excel in the teaching and learning of statistics in secondary schools. This paper demonstrates the ability for Ms Excel to teach almost all the topics in secondary school statistics.
\end{abstract}

Index Terms-Information and communication technology (ICT), Microsoft Excel (MS Excel), spreadsheets, statistics.

\section{INTRODUCTION}

Recent technological developments, offer teachers an additional method for teaching statistics' content and practice. ICT integrated teaching and learning offers a number of advantages and some of these advantages include emphasis on active learning, enrichment of collaborative learning, encouragement of greater student's independence and task-based teaching [1]. ICT integration can be defined as the usage of ICT functioning as an integral or mediated tool to accomplish specific teaching or learning activities to meet certain instructional objectives [2].

ICTs can be defined as a "diverse set of technological tools and resources used to communicate, and to create, disseminate, store, and manage information." These technologies include computers, the Internet, broadcasting technologies (radio and television), and telephony [3].

Studies reveal a number of factors which influence teachers' decisions to use ICT in the classroom. One of the factors is access to quality of software [4]. Schools in developed countries with greater powers of acquisition have easier access to specialised educational quality technologies and can easily integrate ICTs in the teaching and learning curricula. Schools in developing countries on the other hand

Manuscript received October 17, 2014; revised December 19, 2014.

The authors are with Copperbelt University, Zambia (e-mail: nchimunya2009@gmail.com, 1shumba2001@yahoo.com). however do not enjoy these powers and as such it is imperative that they redouble their efforts to prepare themselves to successfully meet the challenge and maximize the opportunities that the use of ICT in education offers [5]. One way in which they could do this is by using readily available tools like spreadsheets.

Users can manipulate numbers by using stored formulas and calculate different outcomes and this capability makes a spreadsheet an ideal tool for the teaching and learning of mathematics. Popular electronic spreadsheet packages include Lotus 1-2-3, Quattro Pro, and Ms Excel. The first electronic spreadsheet though was VisiCalc which appeared in 1979. It was created by Dan Bricklin and Bob Frankston for the Apple II platform. The creators later sold the rights in VisiCalc to Lotus Development Corporation, which developed Lotus 1-2-3 for the IBM PC in 1982. Then came Microsoft Excel, which was developed first for the Apple Macintosh, but which was also the first real Microsoft Windows application [6]-[8].

The use of spreadsheets allows pupils or students to explore solution alternative processes that go beyond symbolic manipulations and provide students with a deeper understanding of concepts embedded in a problem. One unique use of spreadsheets is the ability to interactively model and simulate mathematical situations. Mathematics teachers can use spreadsheets to create experimental environments for discovering mathematical relationships. These experiments can assist pupils or students in their conceptualization of relationships among numerical, graphical and algebraic representations [9].

This paper particularly investigates Ms Excel as a tool for the teaching and learning of mathematics. In this paper we want to illustrate through examples how a spreadsheet such as Ms Excel can be used to construct powerful demonstrations of statistical ideas for teaching purposes.

The rest of the paper is organized as follows: Section II reviews literature on similar work, Section III gives the demonstration of how excel can be used to teach statistics and Section IV concludes the paper.

\section{LITERATURE REVIEW}

Critics of using technology in teaching statistics argue that students have to spend time learning the package and are therefore not concentrating on learning statistics [10]. Some researchers although argue that using a spreadsheet such as Excel can overcome some of these difficulties. In particular, students are more aware of what is happening because they can see the formulae being used and the process of analysis is much more interactive [11]. In addition, most students are 
already familiar with spreadsheets because they are readily available and mostly used in schools.

Petocz and Reid [12] in their study concluded that "statistics is not a 'spectator sport', students learn statistics only if they actually practice statistics through a whole range of statistical activity supported by an appropriate computer package and discussion".

Researchers all over the world emphasise on the usage of specialized statistics packages, such as SPSS or SAS/STAT in the analysis of statistical data. A few teachers in Universities have adopted these specialised packages and use them for teaching quantitative and research methods courses. Statistical software like SPSS allows the user to perform standard statistical tests but do not require the user to understand the calculation process. Students or pupils need to learn to break down problems into manageable chunks to calculate and check intermediate results. It is this ability that makes spreadsheets suitable for education purposes [13].

Warner et al. [14] argues that with charting capabilities and the Analysis ToolPak, which provides a set of statistical analysis tools found in general packages like excel, makes these packages resemble what one would expect from a specialized low-level statistics program. Spreadsheets can thus be used as alternatives to the specialized packages.

Spreadsheets such as Ms Excel also offer a number of advantages as compared to the specialised packages. Nash and Quon [15] identifies the following advantages offered by spreadsheet software to both teachers and students.

1) The widespread use and knowledge of spreadsheet software saves the costs of acquiring, teaching, and learning the mechanics of a new software tool.

2) Such software is often taught and supported by staff other than those who teach statistics.

3) Teachers can prepare templates in advance for students to follow and carry out particular computations.

4) With some exceptions, the spreadsheet calculation paradigm offers immediate updating of results when data are changed.

5) Spreadsheets are a fairly general computational tool, so they can often be "programmed" to perform non-standard calculations.

6) As mentioned, spreadsheet software now offers tools for many common statistical calculations.

7) Spreadsheets are a handy tool for data entry, editing, and manipulation prior to input to a standard statistics package for analysis.

A number of researches have been carried out to investigate the suitability of spreadsheets in the teaching and learning of mathematics and in particular statistics. Most researchers tended to come up with the similar conclusion that the integration of spreadsheets in the teaching and learning of statistics enhances the teaching and the understanding of the subject by many pupils or students. For example, Lee and Soper [16] argued in their paper, that "teaching methods of statistical calculation in geography may be enhanced by using a spreadsheet computer program. The student enters computational formulae in stages, thus gaining an understanding of the calculation process whilst having the actual arithmetic done by computer. The input data, intermediate calculations, and results are laid out in a matrix of rows and columns. They can be inspected and altered to demonstrate various statistical properties."

Warren et al. [17] carried out a study that evaluated a set spreadsheet-based tutorials developed for the statistics portion of an undergraduate psychology research methods course. The evaluation involved a quasi-experimental comparison of spreadsheet-based and traditional classroom teaching methods in a course that gave equal teaching time to both solutions, and took the unique approach of investigating both outcomes-based and attitudinal measures in the same study. The output of the study showed that the spreadsheet-based tutorials improved student outcomes-based performance on an end-of-semester test; it also showed an improvement in student's attitudes towards the course material.

A study was done which looked at the learning activity in middle school which made use of a spreadsheet to mediate students' construction of meanings for statistical conceptions. The study illustrated the integration of computers in teaching and learning statistics as it brings about a cascade of changes in curriculum materials, classroom praxis, and students' ways of learning. The study also looked at the impact of technological tools on teaching and learning statistics by emphasizing how the computer lends itself to supporting cognitive and sociocultural processes. The authors concluded that the use of spreadsheets in the teaching of statistical concepts enhances the understanding of these concepts by students [18].

A further study was done that looked at the use of Excel in the introductory course in statistics in a business school. An attempt was made to identify how business school programs differ, and how the use of Excel to work cases or examples can contribute to a business statistics course that is in keeping with the schools and the instructor's learning objectives. The study presented some ideas for using spreadsheets that may be appropriate in teaching statistics in the business school. The study concluded that the use of Excel together with realistic business examples and exercises can contribute greatly to students understanding of both business and statistics [19].

These and many more researches demonstrate the fact that spreadsheets have more positive sides as tools for the teaching and learning of statistics in Schools. Most of the research done though does not demonstrate how topics in statistics can be delivered using spreadsheets like Ms Excel. This paper fills in this gap by giving specific examples on how basic statistics can be delivered to pupils in secondary schools.

\section{Solving Statistical Problems Using MS EXCEL}

The purpose of this chapter is to show how to solve statistics in Excel. The example below will be used to show how excel can be used to solve statistical problems:

Example: Thirty AA batteries were tested to determine how long they would last. The results to the nearest minute were recorded as follows:

$423,365,387,411,393,394,371,377,389,409,392,408$, $431,401,363,391,405,382,400,381,399,415,428,422$, $396,372,410,419,386,390$

\section{A. How to Create a Frequency Table and a Histogram in Excel}


First, the Data Analysis "toolpak" must be installed if not yet installed. Enter the column of numbers in the spreadsheet that you wish to create the frequency table from. Then enter the column of intervals or "Bin" to be the upper boundary for the classes on the table. Fig. 1 below shows a spreadsheet with these entries.

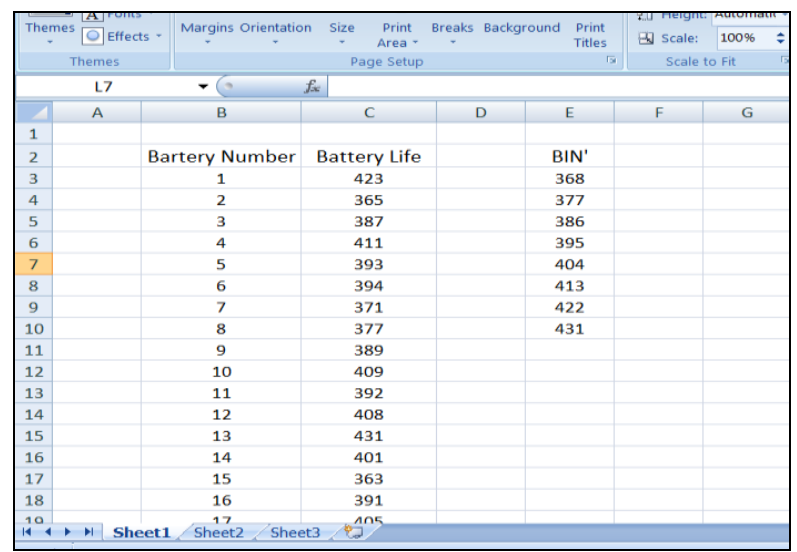

Fig. 1. Spreadsheet showing the column of number and BIN

On the main menu choose Data and then click the Data Analysis tool, then choose Histogram and click OK. Enter the Input Range, in our example above it would be B3:B32, then enter the Bin Range E3:E10. Choose a defined output range on the same spreadsheet. For our example choose the output range as $\mathbf{G 3}$, manually alter the BIN range to represent a range (360-368) instead of just the upper boundary (368), the spreadsheet would look like Fig. 2 below.

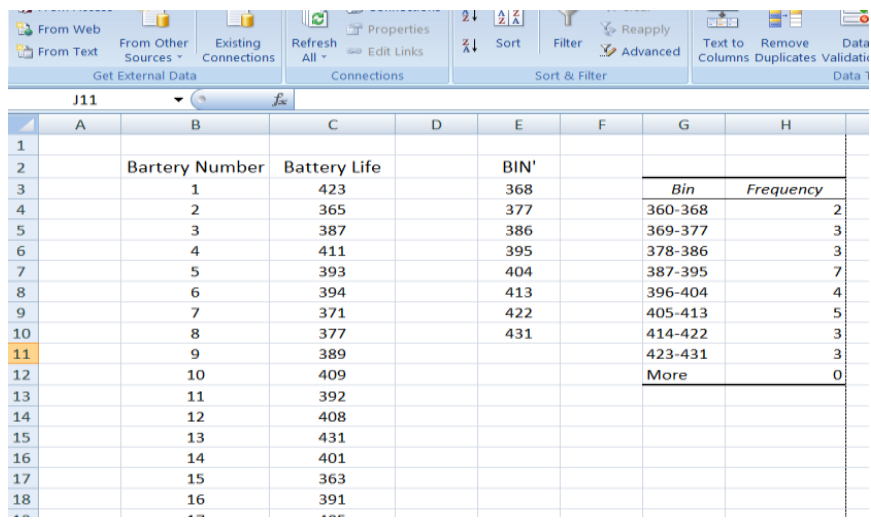

Fig. 2. Showing the generated frequency table in excel.

The next step is to make a histogram from the frequency table in the example above. Select the BIN range and the frequency range, click on insert and choose column chart type and select the chart sub type and click on it. Right click on any of the bars and choose Format Data Series. Choose the Series Options tab, reduce the Gap Width to zero percent and click OK. Dress up the graph by clicking anywhere on the graph and choose layout on the main menu. Use the options that appear to enter a complete descriptive title and axes labels. The completed Histogram is shown in Fig. 3 below.

\section{B. Creating Frequency and Cumulative Frequency Polygons in Excel}

Frequency polygons are made using midpoints on the horizontal axis. So we must start by manually entering the midpoint values for our frequency distribution table in Fig. 1 above. Then select the data in G4 through G11 and H4 through H11. Click on insert and choose Scatter chart type and select "the scattered chart subtype with data points connected by lines" and click it. The completed frequency polygon is shown in Fig. 4 below.

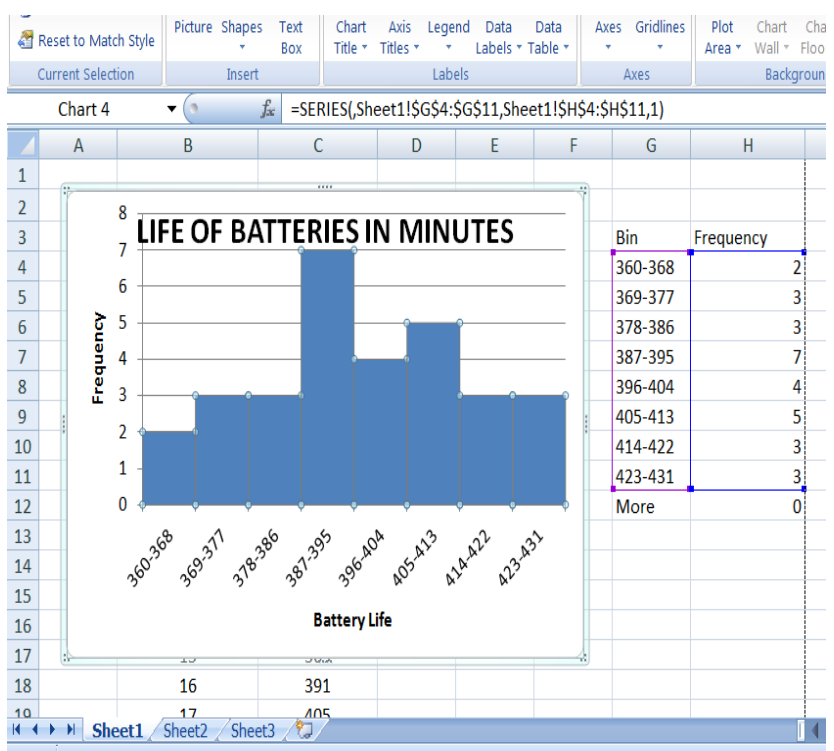

Fig. 3. Showing the generated histogram in excel.

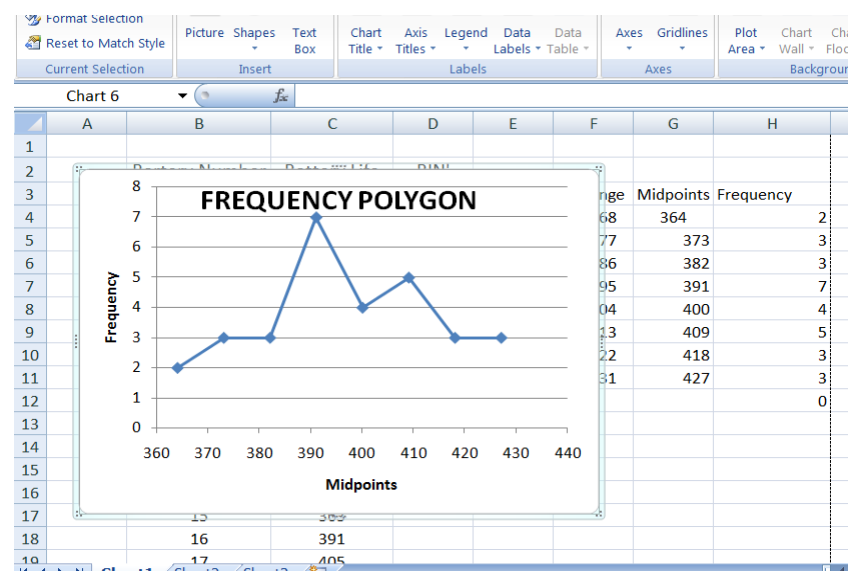

Fig. 4. Showing the generated frequency polygon in excel.

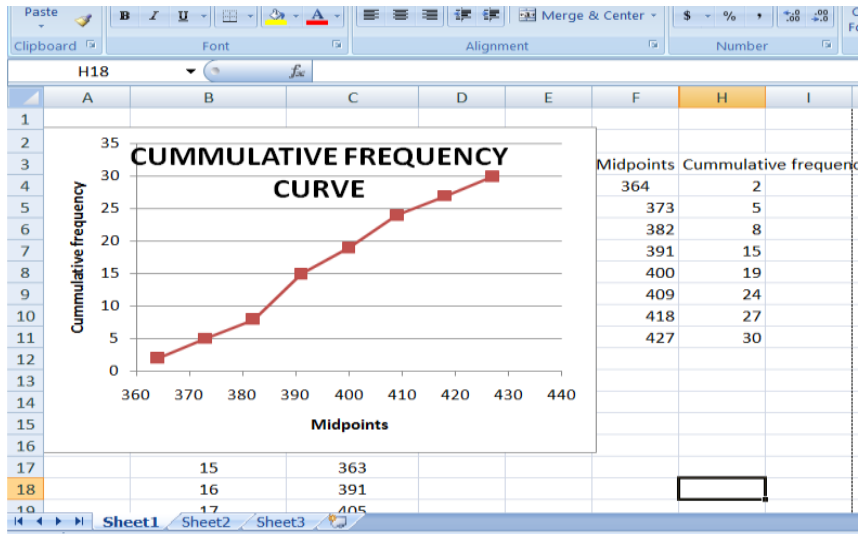

Fig. 5. Showing the generated cumulative frequency curve in excel.

The next step is to construct a cumulative frequency curve or the orgive from the frequency table. Add a Cumulative Frequency column in the frequency table. Place a case in the first cell of the added column. In the example, this would be cell H4. Enter the formula: =FREQUENCY $(\mathrm{C}: \mathrm{C}, \mathrm{D} 3)$. In this formula, the range $\mathbf{C}: \mathbf{C}$ denotes the column that contains all of the scores. The second item, D3, specifies the upper limit of the interval for which we are calculating the cumulative 
frequency. Next, copy the frequency formula from the cell and paste it into the other cells in the Cumulative Frequency Column. To graph the cumulative frequency curve use the same steps used in creating a frequency curve and the completed cumulative frequency curve is shown in Fig. 5 above.

\section{Finding Descriptive Statistics Using Excel}

To find the descriptive statistics, select descriptive statistics from the Data analysis tool. Select the range C3:C32 as the Input Range and select cell $\mathrm{K} 1$ as the Output Range. Make sure Summary statistics is checked and then click ok. The measures will calculated as shown in Fig. 6 below.

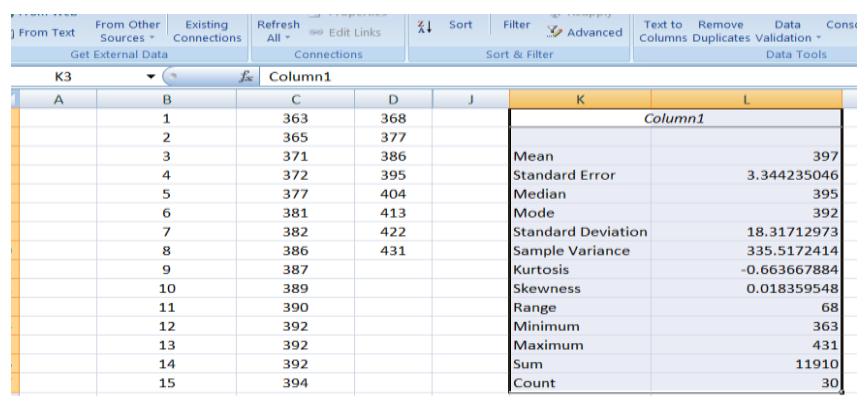

Fig. 6. Showing the generated descriptive statistics in excel.

To find the other descriptive statistics such as the Quartiles, use excel functions such as QUARTILE. It returns the three quartiles of a data set (that is, the first, second and third quartiles). To find the quartiles write = QUARTILE (array, quart), where array is cell range of numeric values for which you want the quartile value and Quart indicates which value to return. If quart is equal to 1,2 or 3, the first, second and third quartile will be calculated respectively. In our example we write =QUARTILE(C3:C32,1), =QUARTILE(C3:C32,2) and =QUARTILE(C3:C32,3) to find the three Quartiles and results are as shown in Fig. 7 below.

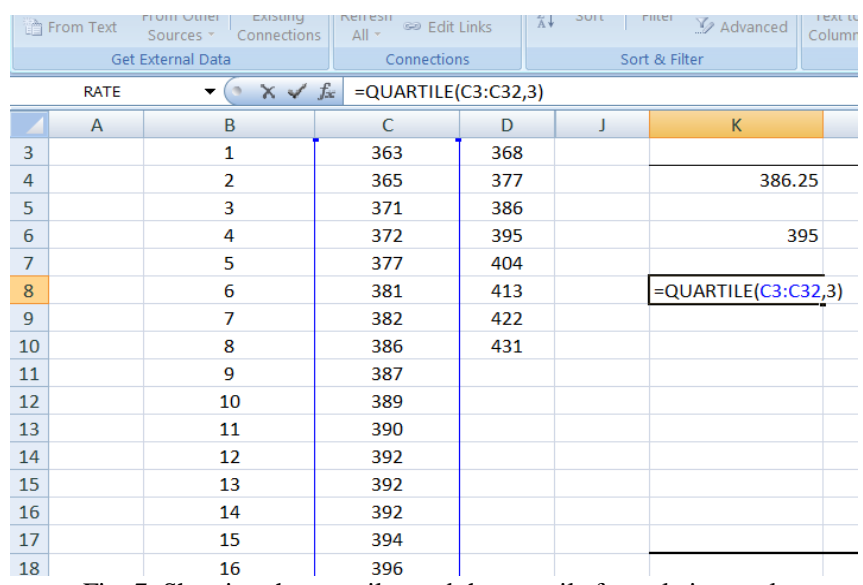

Fig. 7. Showing the quartiles and the quartile formula in excel.

\section{Finding Descriptive Statistics for Grouped Data Using Excel}

This section will consider finding the following descriptive statistics for grouped data: Mode, Mean, Median, Variance and Standard Deviation. Ms Excel does not provide functions for calculating these descriptive statistics for grouped data. The formulas will be manually created. First the frequency table is enhanced to add the following columns: The Lower bounds for each class, the middle values for each class, the cumulative frequency, the product of middle value and frequency and the product of the middle values squared and the frequency.

\section{E. The Mean}

For the mean of a grouped distribution, we assume that all of the data points in a given interval are located at the midpoint of that interval. If $x$ represents the midpoints, $f$ denotes the frequencies, and $n$ is the total number of data points, then, mean $=\frac{\sum(x f)}{n}$. The formula is entered in Excel as shown in Fig. 8 below:

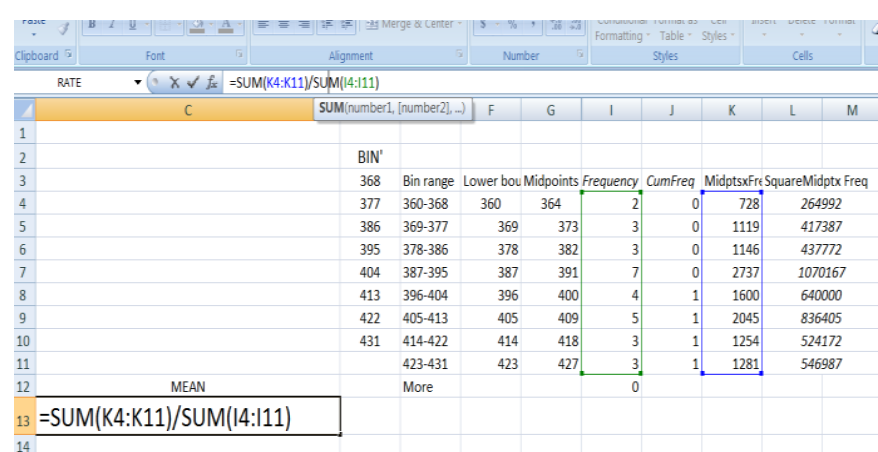

Fig. 8. The defined formula in excel to calculate the mean for grouped data.

\section{F. The Mode}

First we need to determine the modal class which is the class with the highest frequency and then we use the following formula to obtain the mode: $L+\left(\frac{f_{1}-f_{o}}{2 f_{1}-f_{o}-f_{2}}\right) \times h$, where $L$ is the lower limit of the modal class, $f_{1}$ the frequency of the modal class, $f_{o}$ the frequency of the class preceding the modal class, $f_{2}$ the frequency of the class proceeding the modal class and $h$ the class interval. The formula is entered in Excel as shown in Fig. 9 below.

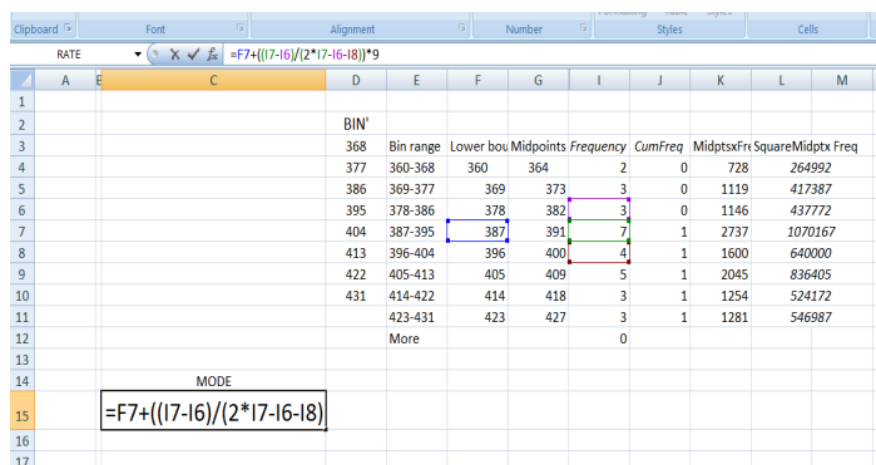

Fig. 9. The defined formula in excel to calculate the mode for grouped data.

\section{G. The Median}

We use the following formula to obtain the median: $L+\left(\frac{\frac{n}{2}-c f}{f}\right) \times h$ , where $L$ is the lower limit of the median class, $c f$ the cumulative frequency before the median class, $f$ the frequency of the median class and $h$ the class interval. The formula is as shown in Fig. 10 below. 


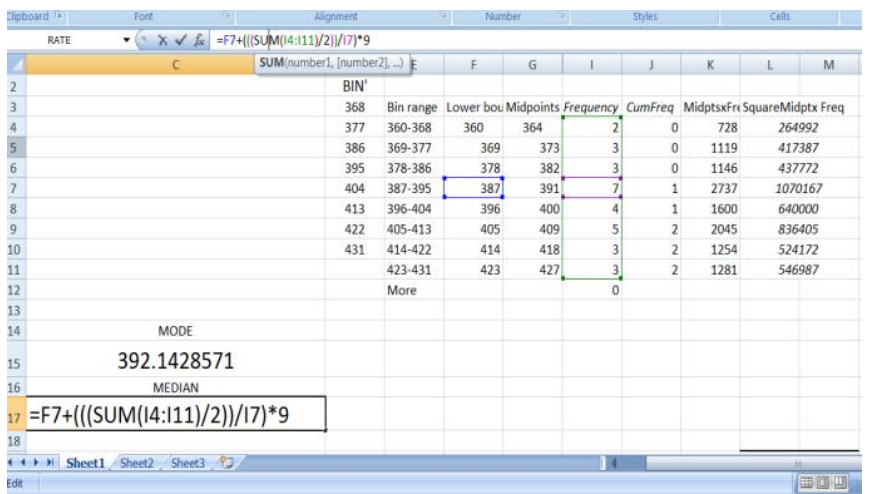

Fig. 10. The defined formula in excel to calculate the median for grouped data.

\section{H. Variance and Standard Deviation}

We use the following formula to obtain the variance: $\frac{\sum f x^{2}-\frac{\left(\sum f x\right)^{2}}{n}}{n-1}$

, where $x$ is the middle value of each class and $n$ is the total frequency. The standard deviation is found by finding the square root of the variance. The formula is entered in Excel as shown in Fig. 11 below.

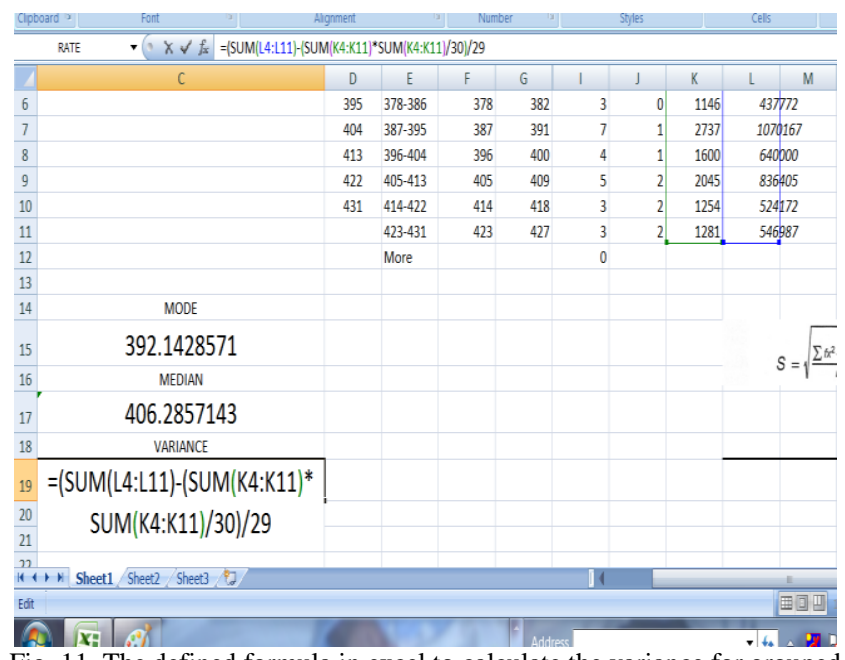

Fig. 11. The defined formula in excel to calculate the variance for grouped data.

Fig. 12 below shows a summary of the descriptive statistics for grouped data obtained by entering formulas in excel. Compared with the descriptive statistics for ungrouped data obtained using the data analysis tool in excel, the values compare very well.

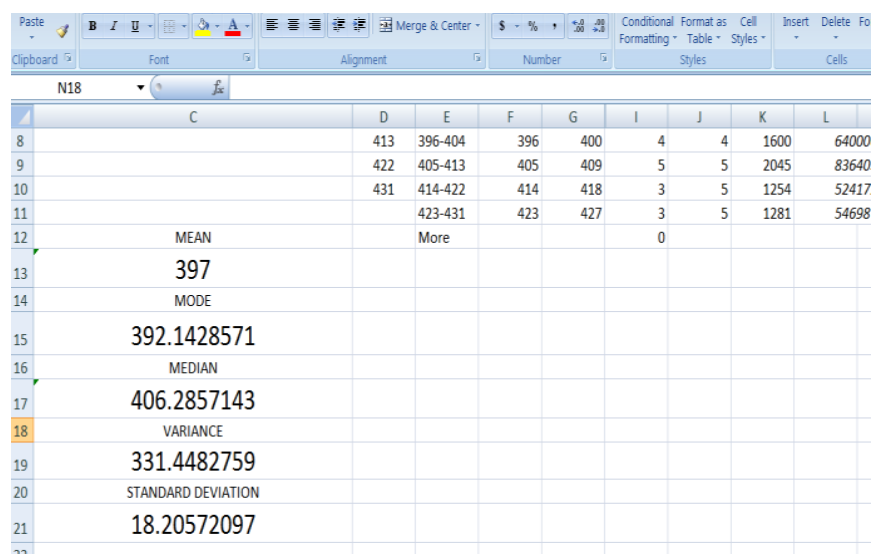

Fig. 12. A summary of the descriptive statistics for grouped data.

\section{CONCLUSION}

This paper has demonstrated the fact that Ms Excel, can be used as a tool to teach subjects like statistics in secondary school mathematics. Researchers have agreed that the use of tools like Excel in the delivery of subjects like statistics enhances the understanding of such subjects by pupils. Ms Excel has an advantage over other tools in that it is widely available and relatively easy to use. This paper demonstrated how Ms Excel can be used to teach basic statistics in secondary schools.

\section{REFERENCES}

[1] R. Basturk, "The effectiveness of computer-assisted instruction in teaching introductory statistics," Journal of Educational Technology \& Society, vol. 8 no. 2, pp. 170-178, 2005.

[2] P. L. Cher and D. Hang, "An activity theory approach to research of ICT integration in Singapore schools," Journal of Computers \& Education, vol. 41, no. 1, pp. 49-53, 2003.

[3] C. Blurton. (September 29, 2014). New directions of ICT-use in education. [Online]. Available: http://www.unesco.org/education/educprog/lwf/dl/edict.pdf

[4] M. Shazia, "Factors affecting teachers' use of information and communications technology," A Review of the Literature, vol. 9, no. 3, pp. 319-342, 2000.

[5] D. Kessy, M. Kaemba, and M. Gachoka, "The reasons for under use of ICT in education: In the context of Kenya, Tanzania and Zambia," in Proc. the Fourth IEEE International Workshop on Technology for Education in Developing Countries, pp. 83-87, Iringa, July 2006.

[6] J. Baker and S. J. Sugden, "Spreadsheets in education - The first 25 years," Spreadsheets in Education Journal (eJSiE), vol. 1, no. 1, 2007.

[7] D. Bricklin. (September 29, 2014). Dan Bricklin's Web Site. [Online]. Available: http://bricklin.com/

[8] E. Trik. History of Microsoft Excel 1978 - 2013. [Online]. Available: http://www.exceltrick.com/others/history-of-excel/

[9] B. C. Peter, "Teaching business statistics with Microsoft Excel," vol. 1, no. 1 , pp. 18-26, 2000.

[10] T. Piazza, "Teaching statistics through data analysis," in Proc. the Inter Conf on Teaching Statistics (ICOTS2), pp. 275-279, 1985.

[11] N. Hunt, "Teaching statistical concepts using spreadsheets," in Proc. the Conf of the Association of Statistics Lecturers in Universities, Teaching Statistics Trust, 1995.

[12] P. Petocz and A. Reid, "Students' experience of learning in statistics," Quaestiones Mathematicae, Supplement 1, pp. 37-45, 2001.

[13] B. S. Jean and P. L. Martin, "Spreadsheets in teaching statistics," Journal of the Royal Statistical Society, Series D (The Statistician), vol. 34 , no. 3, pp. 317-321, 1985.

[14] C. B. Warner and M. A. Meehan, "Microsoft Excel as a tool for teaching basic statistics," Teaching of Psychology, vol. 28, no. 4, pp. 295-298, 2001.

[15] J. C. Nash and K. T. Quon, "Issues in teaching statistical thinking with spreadsheets," Journal of Statistics Education, vol. 4, no. 1, pp. 27-33, 1996.

[16] M. P. Lee and J. B. Soper, "Using spreadsheets to teach statistics in geography," Journal of Geography in Higher Education, vol. 11, no. 1, 1987.

[17] W. King et al., "A quasi-experimental comparison of spreadsheet- and classroom-based statistics tutorials," South African Journal of Psychology, vol. 43, no. 1, pp. 34-45, 2013.

[18] A. R. Neurath and L. J. Stephens, "The effects of using Ms Excel in a high school algebra class," International Journal of Mathematical Education in Science and Technology, vol. 37, no. 6, pp. 721-726, 2006.

[19] D. Ben-Zvi, "The role of tech. in improving students learning," Mathematical Thinking and Learning, vol. 2, no. 1-2, pp. 127-155, 2000.

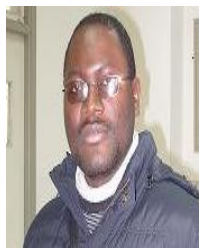

Nchimunya Chaamwe was born in Choma, Zambia 1974. He got his BSc in mathematics at University of Zambia, 2001, the MSc in information technology systems at the University of Strathclyde in Glasgow Scotland, UK., 2003, and the PhD in information and communication engineering at Huazhong University of Science and Technology (HUST) in China, 2011. 
He has worked and working at the Copperbelt University in Zambia in the two capacities: the lecturer from 2003 to 2013, and the senior lecturer from 2013 to Current.

Dr. Nhimunya Chaamwe is a member of the Computer Society of Zambia.

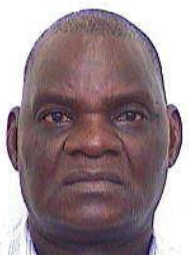

Langstone Shumba was born on April 26, 1955 in Mberengwa District in the Midlands Province in Zimbabwe.

In 2006, he got the certificate in PC maintenance, in University of Zimbabwe. From 1987 to 1988 , he got the certificate in computer engineering (maintenance), from University Control Data Institute (London, UK). And from September 1981 to June
1986, he got his M.Sc. in computer science engineering from University Vinnitsa Polytechnic Institute (USSR). Between 1970 and 1973, he got the GCE'O' Level Certificate (Cambridge) in School Chegato Secondary School in Zimbabwe. He is currently employed by the Copperbelt University (Kitwe, Zambia) as a lecturer in the Computer Science Department.

Mr. Shumba's research interests are in the following areas of computer science: computer security, computer networking and computer architecture. $\mathrm{He}$ is a member of the Computer Society of Zimbabwe. 